\title{
Highly pathogenic avian influenza A virus H5N1 non-structural protein 1 is associated with apoptotic activation of the intrinsic mitochondrial pathway
}

\author{
QIAN BIAN, JING LU, LI ZHANG, YING CHI, YAN LI and HONGXIONG GUO \\ Department of Toxicology and Function Assessment, Jiangsu Provincial Center for \\ Disease Control and Prevention, Nanjing, Jiangsu 210009, P.R. China
}

Received September 29, 2016; Accepted May 25, 2017

DOI: $10.3892 /$ etm.2017.5056

\begin{abstract}
Outbreaks of avian influenza A (H5N1) virus infection have significant health and economic consequences. Non-structural protein 1 (NS1) is an essential virulence factor of the highly pathogenic H5N1 avian influenza virus and of the apoptosis associated with the pathogenesis of H5N1. Previous studies have revealed that the NS1 protein is able to induce apoptosis via an extrinsic pathway. However, it remains unclear whether the intrinsic pathway is also associated with this apoptosis. The present study used a clone of the NS1 gene from avian influenza A/Jiangsu/1/2007 and observed the localization of the NS1 protein and cytochrome $c$ release from mitochondria and the change of mitochondrial membrane potential (MMP) in lung cancer cells. Cytotoxicity was detected using an MTT assay and the number of apoptotic cells was counted using a flow cytometer. Following the isolation of mitochondria, western blotting was performed to compare cytochrome $c$ release from the mitochondria in cells before and after apoptosis. The change of MMP was detected using JC-1 staining. Furthermore, the results reveal that the majority of the NS1 protein was localized in the cell nucleus, and that it may induce apoptosis of human lung epithelial cells. The apoptosis occurred with marked cytochrome $c$ release from mitochondria and a change of the MMP. This indicated that the NS1 protein may be associated with apoptosis induced by an intrinsic mitochondrial pathway.
\end{abstract}

\section{Introduction}

Avian influenza A (H5N1) virus is a highly pathogenic contagious agent that causes severe impairment in poultry

Correspondence to: Professor Hongxiong Guo, Department of Toxicology and Function Assessment, Jiangsu Provincial Center for Disease Control and Prevention, 172 Jiangsu Road, Nanjing, Jiangsu 210009, P.R. China

E-mail: guohongxiong@jscdc.cn

Key words: H5N1, non-structural protein 1, apoptosis, intrinsic mitochondria apoptosis pathway, extrinsic apoptosis pathway and humans, particularly limited person-to-person transmission (1). The cumulative number of confirmed human cases of H5N1 from 14 countries between November 2003 and July 2014 reached 667, 393 of which were fatal according to a report issued by the World Health Organization (2). In total, 47 individuals infected with $\mathrm{H} 5 \mathrm{~N} 1$ were identified in China and $30(63.8 \%)$ succumbed to the H5N1 infection (2). H5N1 causes primary viral pneumonia with rapid progression to lung failure following invasion of epithelial cells in the upper and lower respiratory tracts (3). However, the exact mechanism for elucidating the severity of human H5N1 infection remains unclear. Previously, apoptosis was not only observed in the alveolar epithelial cells of 2 patients who succumbed to $\mathrm{H} 5 \mathrm{~N} 1$ infection, but was also induced by H5N1 in numerous cell types in vivo and in vitro (4-6). These results indicated that apoptosis may be important in $\mathrm{H} 5 \mathrm{~N} 1$ pathogenesis in the human body.

At present, two main apoptotic pathways have been documented; the tumor necrosis factor (TNF) receptor-mediated extrinsic pathway and the intrinsic pathway mediated by mitochondria/cytochrome $c(7)$. The non-structural protein 1 (NS1), which is encoded by the influenza virus NS segment, is able to alter the host response and virulence of the virus in the case of reassortment without prior adaptation $(8,9)$, and is associated with apoptosis regulation in mammalian cells. Previous studies revealed that the H5N1 NS1 protein induced the TNF-mediated extrinsic apoptosis pathway in human alveolar basal epithelial cells (10-13). However, the susceptible cell lines of the various pathogenic avian influenza viruses are different, which causes them to vary in their responses to apoptosis $(14,15)$.

As mentioned above, apoptosis induced by the H5N1 NS1 protein may vary in various cell lines. To further investigate whether the other apoptotic pathways induced by H5N1 NS1 protein exist, H5N1 NS1 protein was used to induce the human lung epithelial cell line, NCI-H292.

\section{Materials and methods}

Construction of an NS1-expressing plasmid. Highly pathogenic avian influenza A/Jiangsu/1/2007 (H5N1) viral RNA was extracted from supernatants of infected cell cultures for 
use as a polymerase chain reaction (PCR) template for amplifying the NS1 gene. Total RNA was extracted from cell lysate using the QIAamp Viral RNA Mini kit (Qiagen, Hilden, Germany) according to the manufacturer's instructions. The full-length NS1 gene was amplified using the SuperScript III One-Step Reverse Transcription-PCR (RT-PCR) system with Platinum Taq High-Fidelity Polymerase (Invitrogen; Thermo Fisher Scientific, Inc., Waltham, MA, USA) from H5N1 virus cDNA. The sense and antisense primers used for NS1 (EU434690) were 5'-GTGCTCGAGATGGATTCCAACACT GTGTCA-3' and 5'-CACGGTACCTCAAACTTCTGACTC AATTGT-3', respectively. The PCR conditions were $95^{\circ} \mathrm{C}$ for $15 \mathrm{~min}$, followed by 34 cycles of $94^{\circ} \mathrm{C}$ for $30 \mathrm{sec}, 58^{\circ} \mathrm{C}$ for $30 \mathrm{sec}$, and $72^{\circ} \mathrm{C}$ for $30 \mathrm{sec}$. The cloning insert was ligated into the pMD18-T vector (D101A; Takara Biotechnology Co., Ltd., Dalian, China) by quick ligase (M2200S; NEB Beijing Ltd., Beijing, China) incubating for $30 \mathrm{~min}$ at room temperature. pMD18-T-NS1 was subcloned into the expression plasmid pXJ40-hemagglutinin (HA) (Invitrogen; Thermo Fisher Scientific, Inc.) using XhoI (D1094A) and KpnI (D1068A) (both from Takara Biotechnology Co., Ltd. Dalian, China) sites to produce the recombinant HA-tagged construct, pXJ40-HA-NS1. The construction of plasmid pXJ40-HA-NS1 followed standard cloning procedures. Competent Escherichia coli TOP10 cells (Invitrogen; Thermo Fisher Scientific, Inc.) were transformed using pXJ40-HA-NS1 plasmids, and the plasmids were amplified and purified using a high-purity plasmid purification kit (Invitrogen; Thermo Fischer Scientific, Inc.). Clones were then screened by restriction enzyme digestion and sequence analysis using the version 3.1 BigDye Terminator ready reaction cycle sequencing kit (Applied Biosystems; Thermo Fischer Scientific, Inc.) according to the manufacturer's instructions.

Cell line culture and transient transfection. Non-small cell lung cancer cell lines, NCI-H1299 and NCI-H292 (Cell Bank; Shanghai Institutes for Biological Sciences, Chinese Academy of Sciences; Shanghai China), were separately grown as a monolayer in Dulbecco's modified Eagle's medium (DMEM) (Invitrogen; Thermo Fischer Scientific, Inc.) supplemented with $10 \%$ fetal bovine serum (Invitrogen Thermo Fischer Scientific, Inc.) at $37^{\circ} \mathrm{C}$ and in a $5 \% \mathrm{CO}_{2}$ incubator. The two cell lines were used for different purposes, NCI-H1299 was used to observe the localization of the NS1 protein in the cell whereas NCI-H292 was used to confirm the extent of apoptosis induced by the NS1 protein.

NCI-H1299 and NCI-H292 were transfected with pXJ40-HA-NS1 or control plasmids (pXJ40-HA-vector; Invitrogen; Thermo Fischer Scientific, Inc.) using Lipofectamine 2000 reagent according to the manufacturer's instructions (Invitrogen; Thermo Fisher Scientific, Inc). After $4 \mathrm{~h}$, Lipofectamine 2000-DNA complexes were removed, and the cell culture DMEM medium was replaced with fresh DMEM with or without $0.025 \mathrm{nM}$ staurosporine (STS; Sigma-Aldrich; Merk KGaA, Darmstadt, Germany) at $37^{\circ} \mathrm{C}$ for $24 \mathrm{~h}$. Cells were collected after $24 \mathrm{~h}$, washed with phosphate-buffered saline (PBS) and trypsinized with $0.125 \%$ trypsin/EDTA solution.
Immunofluorescence staining. NCI-H1299 cells were fixed in $4 \%(\mathrm{w} / \mathrm{v})$ paraformaldehyde at room temperature for $30 \mathrm{~min}$ and permeablized in $0.5 \%$ (w/v) Triton X-100, followed by incubation with primary and secondary antibodies for $1 \mathrm{~h}$ at room temperature sequentially. Anti-HA serum (AH158; Beyotime Institute of Biotechnology, Haimen, China) with 1:200 was used for the control and Alexa 488-conjugated secondary antibody (1:500, A-11017; Invitrogen; Thermo Fisher Scientific, Inc.) were used to probe for the NS1 protein at room temperature for $1 \mathrm{~h}$. Following protein staining, anti-cytochrome c monoclonal antibody (1:1,000, BD556432; BD Biosciences, Franklin Lakes, NJ, USA) and Alexa 555-conjugated secondary antibody (1:500, A-21427; Invitrogen; Thermo Fisher Scientific, Inc.) were utilized to probe the NCI-H1299 cellular morphology. Finally, 4',6-diamidino-2-phenylindole (DAPI, 1:1,000, D1306; Invitrogen; Thermo Fisher Scientific, Inc.) was used to dye the cell nucleus at room temperature for $1 \mathrm{~h}$. Triple-fluorescence stained cells were observed with a confocal microscope at a high-power magnification of x100 (FV10-ASW, version 01.07.03.00; Olympus Corporation, Tokyo, Japan).

Cell viability assay. MTT cell viability assays were performed according to the manufacturer's instructions (Sigma-Aldrich; Merck KGaA). Briefly, $20 \mu 15 \mathrm{mg} / \mathrm{ml} \mathrm{MTT} \mathrm{was} \mathrm{added} \mathrm{to}$ the culture medium. Following incubation at $37^{\circ} \mathrm{C}$ for $3 \mathrm{~h}$, $100 \mu \mathrm{l}$ acidic isopropanol $(0.1 \mathrm{nM} \mathrm{HCl}$ in acidic isopropanol) was added to the NCI-H292 cells and then the absorbance of each sample was measured at $570 \mathrm{~nm}$ with an automated plate reader (SpectraMax Paradigm; Molecular Devices, LLC, Sunnyvale, CA, USA) compared to the same number of control cells.

Flow cytometric analysis. In order to determine the apoptotic rate, $\sim 1 \times 10^{6} \mathrm{NCI}-\mathrm{H} 292$ cells $/ \mathrm{ml}$ were stained with fluorescein isothiocyanate (FITC)-conjugated Annexin V and propidium iodide (556547, Annexin V-FITC apoptosis detection kit, BD Pharmingen; BD Biosciences) in a volume of $100 \mu \mathrm{l}$ on ice for $30 \mathrm{~min}$ in the dark. The cells were washed 3 times with PBS. Finally, $400 \mu$ l binding buffer was added to the cells. Additionally, the mixture was analyzed with a BD FACSCalibur flow cytometer and the percentage of apoptosis of 10,000 cells was determined. The data were analyzed using BD CellQuest ${ }^{\mathrm{TM}}$ Pro software (version 5.2.1; BD Biosciences) and the percentage cells with apoptosis per group were calculated.

Determination of mitochondrial membrane potential $(M M P)$. At $24 \mathrm{~h}$ following transfection, the culture medium of the NCI-H292 cells was replaced with DMEM that did not include phenol red and was supplemented with $5 \mathrm{mg} / \mathrm{l} \mathrm{JC}-1$ dye (Beyotime Institute of Biotechnology) in the dark for $20 \mathrm{~min}$ at $37^{\circ} \mathrm{C}$. Subsequently, the cells were washed twice with PBS and placed in fresh medium without serum. Finally, MMP was analyzed by calculating the ratio of fluorescence intensity at $555-488 \mathrm{~nm}$ in triplicate.

Mitochondria isolation and calculation of cytochrome $c$ release. Cytosolic and mitochondrial isolation are performed as described by Cheng et al (16). The percentage of cytochrome $c$ 
A PXJ40-HA-NS1 B

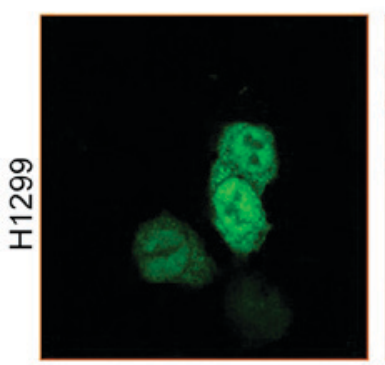

Cyto $c$

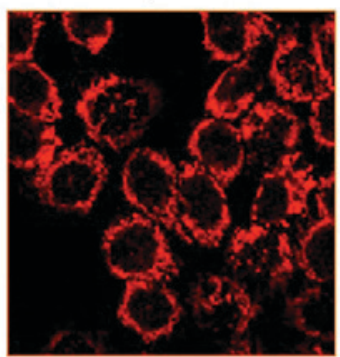

$\mathrm{C}$

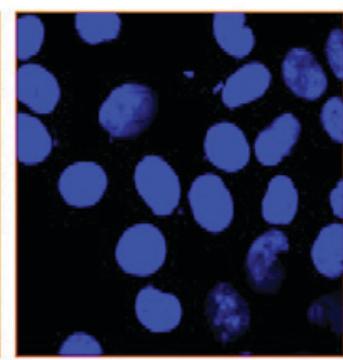

D Merged

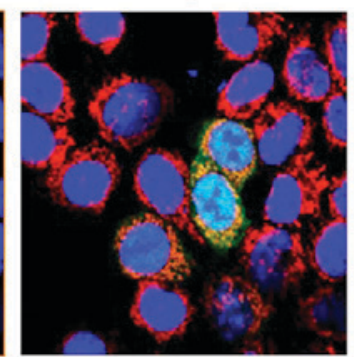

Figure 1. Analysis of the expression and localization of the NS1 protein in the H1299 cell line via immunofluorescence and confocal microscopy (magnification, x100).pXJ40-HA-NS1 transfected H1299 cells were incubated with (A) anti-HA serum and Alexa 488 conjugated secondary antibody, (B) anti-cytochrome $c$ monoclonal antibody and Alexa 555 conjugated secondary antibody and (C) DAPI dye. (D) A merged image is also presented. NS1, non-structural protein 1; HA, hemagglutinin; DAPI, 4',6-diamidino-2-phenylindole; cyto $c$, cytochrome $c$.

release was calculated using the following formula: The percentage of cytochrome $c$ release that is equal to the amount of cytochrome $c$ in the mitochondrial supernatant/the total amount of cytochrome $c$ in mitochondrial supernatant and pellet.

Western blot analysis. Monolayers of cells transfected with DNA or untransfected cells were lysed with ice-cold lysis buffer (150 mM Tris-HCl, pH 8.0,50 mM NaCl, 1 mM EDTA, $0.5 \%$ Nonidet P-40, 1 tablet Complete Mini protein inhibitor mixture $/ 10 \mathrm{ml}$ buffer and $0.7 \mu \mathrm{g} / \mathrm{ml}$ pepstatin), and the total protein concentration was determined using the bicinchoninic acid assay. A total of $7 \mu \mathrm{l}$ proteins with equivalent concentrations $(1 \mu \mathrm{g} / \mu \mathrm{l})$ were heated for $5 \mathrm{~min}$ at $100^{\circ} \mathrm{C}$ in lysis buffer containing $\beta$-mercaptoethanol, and then resolved using $4-20 \%$ SDS-PAGE. The proteins were then transferred onto a polyvinylidene difluoride membrane and blocked with $1 \%$ powdered skimmed milk in Tris-buffered saline with $0.1 \%$ Tween-20 for $1 \mathrm{~h}$ at room temperature. Anti-cytochrome $c$ MAb mouse antibody (1:200, BD556432; BD Biosciences) was then used to probe for cytochrome $c$ overnight at $4^{\circ} \mathrm{C}$. The membrane was also probed for GADPH (1:1,000, A2066; Sigma-Aldrich; Merck KGaA) was used as the loading control. Membranes were subsequently washed with $150 \mathrm{mM}$ PBS and incubated for $1 \mathrm{~h}$ at $4^{\circ} \mathrm{C}$ in $10 \%$ dried milk in PBS. Membranes were washed 5 times with PBS and subsequently incubated for $1 \mathrm{~h}$ at room temperature with anti-mouse immunoglobulin $\mathrm{G}$ horseradish peroxidase-conjugated secondary antibody $(1: 1,000$, ZB2307; ZSGB BIO; OriGene Technologies, Inc., Rockville, MD, USA)., followed by visualization of positive bands with the Pierce (Thermo Fisher Scientific, Inc.) enhanced chemiluminescence procedure using Kodak BioMax film. Blots were scanned and the protein ratios were calculated using the PDQuest program (version 7.4.0; Bio-Rad Laboratories, Inc.). The results shown are representative of 3independent experiments.

Statistical analysis. In the cell viability assays and MMP experiments, all data were expressed as the mean \pm standard deviation. Statistical significance among groups was assessed using one-way analysis of variance and the Tukey test. $\mathrm{P}<0.05$ was considered to indicate a statistically significant difference. Finally, statistical analyses were performed using GraphPad Prism 5.0 (GraphPad Software, Inc., La Jolla, CA, USA).

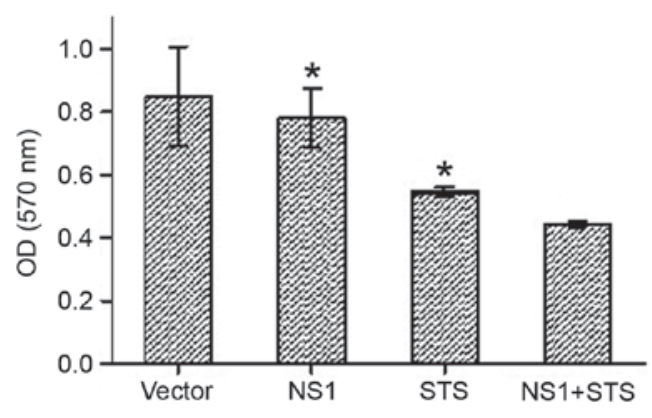

Figure 2. Cytotoxicity detection by MTT assay on NCI-H292 cells transfected by pXJ40-HA-NS1 for $24 \mathrm{~h}$. Cells transfected with empty vector or pXJ40-HA-NS1 and treated with or without STS $(0.025 \mathrm{nmol})$ were used. ${ }^{*} \mathrm{P}<0.05$ vs. NS1 + STS group. HA, hemagglutinin; NS1, non-structural protein 1; STS, staurosporine; OD, optical density.

\section{Results}

Location of H5N1 NS1 protein in NCI-H1299 cells. The plasmid pXJ40-HA-NS1 was transfected into NCI-H1299 cells, and the expression and location of the NS1 protein was monitored. The plasmid pXJ40-NS1-HA includes the full-length NS gene cloned from influenza H5N1. As demonstrated in Fig. 1, the NS1 protein began to express $24 \mathrm{~h}$ following transfection (Fig. 1A), and predominantly remained in the nucleus (Fig. 1B-D).

H5N1 NS1 protein induced apoptosis in NCI-H292 cells. Previous studies have revealed that the NS1 protein of H5N1 is able to induce apoptosis in A549 cells and human airway epithelial cells $(17,18)$. The present study attempted to clarify whether the expression of NS1 was sufficient to induce apoptosis with or without STS, which was a confirmed apoptosis inducer, and STS (0.025 nM) was used as a positive control. Initially, NS1 protein and STS were used to induce cytotoxicity, and the cytotoxicity was then detected under various conditions using the MTT assay. Compared with cells transfected with empty vector, the viability of NS1-transfected cells was markedly lower (Fig. 2). However, the addition of STS significantly decreased the viability of the cells compared with NS1-transfected cells and STS-treated cells, which suggested an increase in cytotoxicity $(\mathrm{P}<0.05)$.

To further confirm cell apoptosis, which is associated with NS1 protein expression, the NCI-H292 cells expressing 

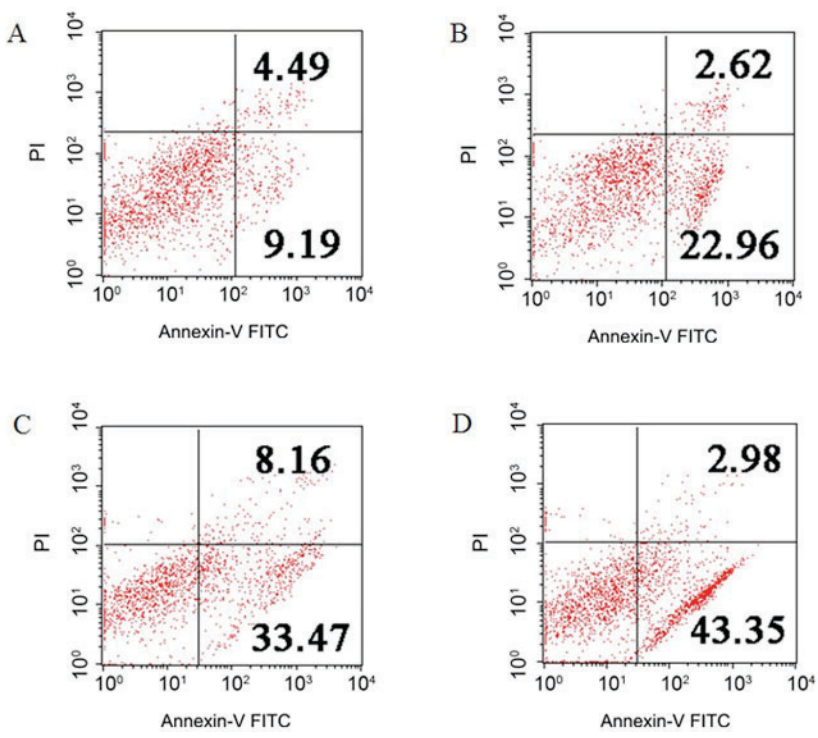

Figure 3. Identification of apoptosis by the FCM assay of Annexin V/PI double staining. FCM assay is represented by dot plot diagrams that demonstrate the typical apoptotic cell population with Annexin-V FITC and PI staining. (A) NCI-H292 cells transfected with empty pXJ40 vectors; (B) NCI-H292 cells transfected with pXJ40-HA-NS1; (C) NCI-H292 cells treated with $0.025 \mathrm{nmol}$ STS and (D) NCI-H292 cells transfected with pXJ40-HA-NS1 and treated with $0.025 \mathrm{nmol}$ STS. The lower left quadrants of the panels show the intact viable cells, which were negative for Annexin V and PI staining; whereas the lower right quadrants represent apoptotic cells, which were positive for Annexin V and negative for PI. FCM, flow cytometry; PI, propidium iodide; FITC, fluorescein isothiocyanate; HA, hemagglutinin; NS1, non-structural protein 1; STS, staurosporine.

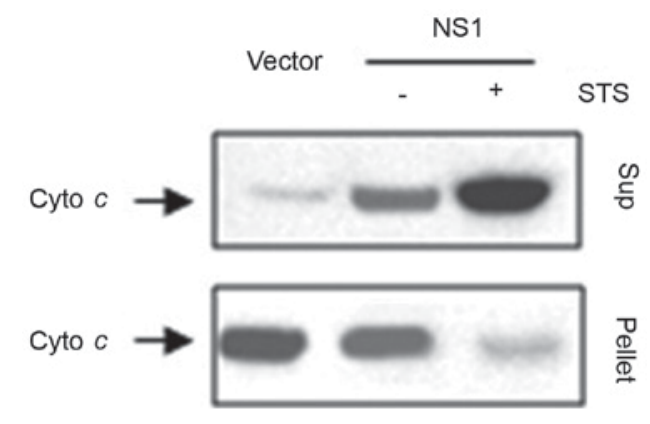

Figure 4. Cytochrome $c$ release from the mitochondria to the cytosol detected by western blotting in NCI-H292 cells transfected with empty vector or pXJ40-hemagglutinin-NS1 and treated with or without STS $(0.025 \mathrm{nmol})$ for $24 \mathrm{~h}$. STS, staurosporine; cyto $c$, cytochrome $c$; NS1, non-structural protein 1. Sup, suspension.

NS1 protein were stained with Annexin V-FITC and PI, and detected using flow cytometry (Fig. 3). Compared with the empty vector-transfected cells $\left(9.19 \%\right.$ Annexin $\mathrm{V}^{+} / \mathrm{PI}^{-}$, indicating early apoptosis, and $4.49 \%$ Annexin $\mathrm{V}^{+} / \mathrm{PI}^{+}$, indicating late apoptosis), the NS1-transfected cells were $22.96 \%$ Annexin $\mathrm{V}^{+} / \mathrm{PI}-$ and $2.62 \%$ Annexin $\mathrm{V}^{+} / \mathrm{PI}^{+}$. Following treatment with STS, NS1-transfected cells were $43.35 \%$ Annexin $\mathrm{V}^{+} / \mathrm{PI}$ and $2.98 \%$ Annexin $\mathrm{V}^{+} / \mathrm{PI}^{+}$, whereas empty vector-transfected cells were $33.47 \%$ Annexin $\mathrm{V}^{+} / \mathrm{PI}^{-}$and $8.16 \%$ Annexin $\mathrm{V}^{+} / \mathrm{PI}^{+}$. Compared with the empty vector-transfected group, the number of apoptotic cells in the NS1-plasmid transfected group was significantly increased $(\mathrm{P}<0.05)$. Following combinational treatment of NS1 and STS, the number of apoptotic cells was

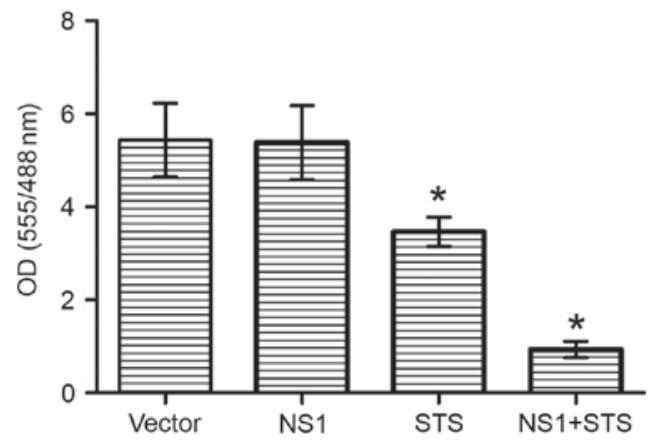

Figure 5. Mitochondrial membrane potential detection on NCI-H292 cells transfected with empty vector or pXJ40-hemagglutinin-NS1 and treated with or without STS $(0.025 \mathrm{nmol})$ for $24 \mathrm{~h}$. ${ }^{*} \mathrm{P}<0.05 \mathrm{vs}$. NS1 group. STS, staurosporine; NS1, non-structural protein 1; OD, optical density.

significantly increased compared with the NS1 group $(\mathrm{P}<0.05)$. These results indicate that NS1 is associated with the apoptosis of NCI-H292 cells, and that the effect of apoptosis induced by NS1 protein may be enhanced by STS.

Involvement of cytochrome c in NSI-induced apoptosis. As demonstrated in Fig. 4, NS1 together with STS induced the release of cytochrome $c$ from the mitochondria to the cytosol. It is known that cytochrome $c$ release from the mitochondria to the cytosol is a signal for apoptosis (19). This implies the possibility of NS1 protein being associated with apoptosis by activating the intrinsic pathway through cytochrome $c$. In order to confirm this, MMP was initially detected by JC-1 staining. Normal cells stained with JC-1 emitted mitochondrial orange-red fluorescence with a slight green fluorescence, whereas the ratio of orange-red and green fluorescence was inverted when cell apoptosis was induced by NS1 protein. As shown in Fig. 5, in comparison with the empty vector transfected cells group, NS1 transfected cells significantly initiated apoptosis with STS (the ratio of fluorescence intensity at $555 / 488 \mathrm{~nm}$ was $\sim 1.00 ; \mathrm{P}<0.05$ ). However, without STS treatment, the NS1-expressing NCI-H292 cells have no significant difference with the empty vector.

To further corroborate the effect of the mitochondria/cytochrome $c$ on the intrinsic apoptosis pathway inducted by the NS1 protein, western blotting was performed to detect the expression of cytochrome $c$ in mitochondrial pellets and supernatants. The majority of cytochrome $c$ expressed in mitochondrial pellets was isolated from the cells transfected with empty vector. It is noteworthy that the amount of cytochrome $c$ was markedly decreased in the mitochondrial pellets following NS1-transfection for $24 \mathrm{~h}$, whereas the amount of cytochrome $c$ was markedly increased in the mitochondrial supernatant (Fig. 4). These results indicate that NS1 may trigger the release of cytochrome $c$ from mitochondria and that this effect is enhanced by STS.

\section{Discussion}

The results of the present study indicate that the NS1 protein of the H5N1 highly pathogenic avian influenza A virus strain is associated with apoptotic activation of NCI-H1299 through the intrinsic mitochondrial pathway. The MTT assay and flow 
cytometric analysis revealed that the NS1 protein-induced apoptosis of NCI-H1299 cells and its activity may be enhanced by STS. Additionally, the NS1 protein together with STS was able to cause the release of cytochrome $c$ from the mitochondria to the cytosol, and the change of MMP. During the intrinsic mitochondrial apoptosis process, the permeabilization of the mitochondrial outer membrane is the critical step, which results in the release of several apoptogenic factors from the intermembrane space of mitochondria (20-22). Cytochrome $c$ is one of these factors, which binds to the adaptor apoptotic peptidase-activating factor 1 that subsequently recruits cytosolic pro-caspase-9 into a heptameric apoptosome (23). The intrinsic mitochondrial apoptosis pathway is one of the major pathways during viral pathogenesis that include human immunodeficiency virus and severe acute respiratory syndrome coronavirus $(24,25)$.

Although Zhang et al (10) demonstrated that the H5N1 NS1 protein induced caspase-dependent apoptosis in human alveolar basal epithelial cells, Neuman et al (20) revealed that the activation of caspase is the common end event during apoptosis. Therefore, the study by Zhang et al (10) did not have enough evidence to support that the NS1 of H5N1 induced apoptosis through an extrinsic pathway. In the present study, the efficiency of NS1 in inducing apoptosis is lower than that of STS, however, the increasing synergistic effect on inducing apoptosis between STS and NS1 were observed. In addition, in the present study, the plasmid with the NS1 gene was transfected with Lipofectamine 2000 and the transfecting efficiency was lower than the penetrating ability of STS. At this point, although the capacity of inducing apoptosis of NS1 is weaker than STS in the present study, the importance of each one needs to be investigated further. Previous studies have revealed that the NS1 protein of influenza A viruses is a multifunctional viral protein that modulates the virus replication cycle and viral protein synthesis, and HA and neuropilin-1 (NP1) of H5N1 also induce apoptosis of airway epithelial cells $(26,27)$. In vivo, there is a need to determine whether the synergetic effect on inducing apoptosis by NP1, nucleoprotein and HA exists. In particular, these proteins induce apoptosis by either sharing the same pathway or not.

In conclusion, the results of the present study reveal that the intrinsic mitochondrial apoptosis pathway is associated with the apoptosis induced by the NS1 protein of H5N1. Therefore, this may be a novel mechanism in the ability of highly pathogenic avian influenza A virus H5N1 causing severe impairment in humans.

\section{Acknowledgements}

The present study was supported by the National Natural Science Foundation of China (grant no. NSFC 81302466), Jiangsu Provincial 'Twelfth five-year plan' Key Provincial Talents Program (grant no. H201118) and Project 333 Talents in Jiangsu (grant no. BRA2015490).

\section{References}

1. Wang H, Feng Z, Shu Y, Yu H, Zhou L, Zu R, Huai Y, Dong J, Bao C, Wen L, et al: Probable limited person-to-person transmission of highly pathogenic avian influenza A (H5N1) virus in China. Lancet 371: 1427-1434, 2008.
2. http://www.who.int/influenza/human_animal_interface/2017_02 14_tableH5N1.pdf?ua=1. Accessed October 2, 2014.

3. Nicholls JM, Chan MC, Chan WY, Wong HK, Cheung CY, Kwong DL, Wong MP, Chui WH, Poon LL, Tsao SW, et al: Tropism of avian influenza A (H5N1) in the upper and lower respiratory tract. Nat Med 13: 147-149, 2007.

4. Tripathi S, Batra J, Cao W, Sharma K, Patel JR, Ranjan P, Kumar A, Katz JM, Cox NJ, Lal RB, et al: Influenza A virus nucleoprotein induces apoptosis in human airway epithelial cells: Implications of a novel interaction between nucleoprotein and host protein clusterin. Cell Death Dis 28: e562, 2013.

5. Daidoji T, Koma T, Du A, Yang CS, Ueda M, Ikuta K and Nakaya T: H5N1 avian influenza virus induces apoptotic cell death in mammalian airway epithelial cells. J Viro 82: 11294-11307, 2008.

6. Sarmento L, Wasilenko J and Pantin-Jackwood M: The effects of NS gene exchange on the pathogenicity of H5N1 HPAI viruses in ducks. Avian Dis 54 (Suppl 1): S532-S537, 2010.

7. Herold S, Ludwig S, Pleschka S and Wolff T: Apoptosis signaling in influenza virus propagation, innate host defense, and lung injury. J Leukoc Biol 92: 75-82, 2012.

8. Lam WY, Tang JW, Yeung AC, Chiu LC, Sung JJ and Chan PK: Avian influenza virus a/Hk/483/97(H5N1) NS1 protein induces apoptosis in human airway epithelial cells. J Virol 82: 2741-2751, 2008.

9. Lam WY, Yeung AC and Chan PK: Apoptosis, cytokine and chemokine induction by non-structural 1 (NS1) proteins encoded by different influenza subtypes. Virol J 8: 554, 2011.

10. Zhang C, Yang Y, Zhou X, Yang Z, Liu X, Cao Z, Song H, He Y and Huang P: The NS1 protein of influenza a virus interacts with heat shock protein hsp90 in human alveolar basal epithelial cells: Implication for virus-induced apoptosis. Virol J 8: 181, 2011.

11. Ehrhardt C, Wolff T, Pleschka S, Planz O, Beermann W, Bode JG, Schmolke M and Ludwig S: Influenza A virus NS1 protein activates the Pi3k/Akt pathway to mediate antiapoptotic signaling responses. J Virol 81: 3058-3067, 2007.

12. Mukherjee S, Majumdar S, Vipat VC, Mishra AC and Chakrabarti AK: Non structural protein of avian influenza a (H1N1) virus is a weaker suppressor of immune responses but capable of inducing apoptosis in host cells. Virol J 9: 149, 2012.

13. Yang N, Hong X, Yang P, Ju X, Wang Y, Tang J, Li C, Fan Q, Zhang F, Chen Z, et al: The 2009 pandemic a/wenshan/01/2009 H1N1 induces apoptotic cell death in human airway epithelial cells. J Mol Cell Biol 3: 221-229, 2011.

14. Liu B, Meng D, Wei T, Zhang S, Hu Y and Wang M: Apoptosis and pro-inflammatory cytokine response of mast cells induced by influenza A viruses. PLoS One 9: e100109, 2014.

15. Hui KP, Li HS, Cheung MC, Chan RW, Yuen KM, Mok CK, Nicholls JM, Peiris JS and Chan MC: Highly pathogenic avian influenza H5N1 virus delays apoptotic responses via activation of STAT3. Sci Rep 6: 28593, 2016.

16. Cheng EH, Wei MC, Weiler S, Flavell RA, Mak TW, Lindsten T and Korsmeyer SJ: Bcl-2, Bcl-X(L) sequester BH3 domain-only molecules preventingbax- and BAK-mediated mitochondrial apoptosis. Mol Cell 8: 705-711, 2001.

17. Zhang C, Yang Y, Zhou X, Liu X, Song H, He Y and Huang P: Highly pathogenic avian influenza A virus H5N1 NS1 protein induces caspase-dependent apoptosis in human alveolar basal epithelial cells. Virol J 7: 51, 2010.

18. Zhang CF, Jiang SW, Zhu HQ, Yang YT, Yang ZX, Xu L, Zhao LX, Zhou XW and Huang PT: Cloning NS1 gene of H5N1 avian influenza virus and apoptosis induced by it in human pulmonary carcinoma cell line A549. Bing Du Xue Bao 23: 360-365, 2007 (In Chinese).

19. Lin Y, Shi R, Wang X and Shen HM: Luteolin, a flavonoid with potentials for cancer prevention and therapy. Curr Cancer Drug Targets 8: 634-646, 2008.

20. Neumann S, El Maadidi S, Faletti L, Haun F, Labib S, Schejtman A, Maurer U and Borner C: How do viruses control mitochondria-mediated apoptosis? Virus Res 209: 45-55, 2015.

21. Li Y, Li J, Huang H, Yang M, Zhuang D, Cheng X, Zhang H and Fu X: Microcystin-LR induces mitochondria-mediated apoptosis in human bronchial epithelial cells. Exp Ther Med 12: 633-640, 2016.

22. Firsov AM, Kotova EA, Orlov VN, Antonenko YN and Skulachev VP: A mitochondria-targeted antioxidant can inhibit peroxidase activity of cytochrome c by detachment of the protein from liposomes. FEBS Lett 590: 2836-2843, 2016. 
23. Lauber K, Appel HA, Schlosser SF, Gregor M, Schulze-Osthoff K and Wesselborg S: The adapter protein apoptotic protease-activating factor-1 (Apaf-1) is proteolytically processed during apoptosis. J Biol Chem 276: 29772-29781, 2001.

24. Deniaud A, Brenner C and Kroemer G: Mitochondrial membrance permeabilization by HIV-1 Vpr. Mitochondrion 4: 223-233, 2004.

25. Tan YX, Tan TH, Lee MJ, Tham PY, Gunalan V, Druce J, Birch C, Catton M, Fu NY, Yu VC and Tan YJ: Induction of apoptosis by the severe acure respiratory syndrome coronavirus 7 a protein is dependent on tis interaction with the Bcl-xL protein. J Virol 81 6346-6355, 2007.
26. Thulasi Raman SN and Zhou Y: Networks of host factors that interact with NS1 protein of influenza A virus. Front Microbiol 7: 654, 2016.

27. Matsuoka Y, Matsumae H, Katoh M, Eisfeld AJ, Neumann G, Hase T, Ghosh S, Shoemaker JE, Lopes TJ, Watanabe T, et al: A comprehensive map of the influenza A virus replication cycle. BMC Syst Biol 7: 97, 2013. 